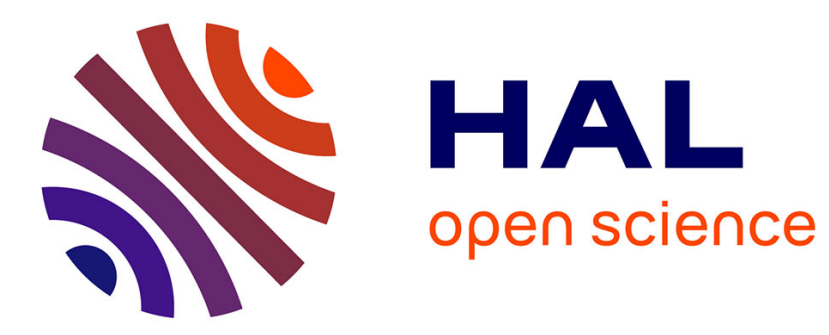

\title{
Le manque d'évaluation des technosciences. Le cas des biotechnologies, des pesticides et de la santé humaine
}

Frédérick Lemarchand, Gilles-Eric Seralini

\section{To cite this version:}

Frédérick Lemarchand, Gilles-Eric Seralini. Le manque d'évaluation des technosciences. Le cas des biotechnologies, des pesticides et de la santé humaine. Cahiers, Droit, Sciences \& Technologies, 2010, Open science et marchandisation des connaissances, 3, pp.231-244. 10.4000/cdst.240 . hal-02303800

HAL Id: hal-02303800

https://hal-normandie-univ.archives-ouvertes.fr/hal-02303800

Submitted on 2 Oct 2019

HAL is a multi-disciplinary open access archive for the deposit and dissemination of scientific research documents, whether they are published or not. The documents may come from teaching and research institutions in France or abroad, or from public or private research centers.
L'archive ouverte pluridisciplinaire HAL, est destinée au dépôt et à la diffusion de documents scientifiques de niveau recherche, publiés ou non, émanant des établissements d'enseignement et de recherche français ou étrangers, des laboratoires publics ou privés. 


\title{
Le manque d'évaluation des techno- sciences. Le cas des biotechnologies, des pesticides et de la santé humaine.
}

\author{
Frederick Lemarchand \\ Gilles-Eric Seralini
}

\begin{abstract}
Parmi les biotechnologies agricoles, les «OGM à pesticides» désignent les plantes ayant subi une modification génétique destinée à les rendre soit tolérantes à un pesticide, soit à les faire produire un pesticide. Contre toute attente, cette innovation technologique mise sur le marché depuis une quinzaine d'années n'a jamais atteint, en Europe, ni le succès ni le seuil de rentabilité escompté par les industriels de la biologie et de l'agro-alimentaire concernés (en premier lieu, la firme américaine Monsanto). Les OGM alimentaires sont encore produits à $98 \%$ sur le continent américain. L'étiquetage obligatoire n'est par contre pas en vigueur aux États-Unis, où le gouvernement s'oppose le plus à cette idée, malgré une demande de la majorité de la population. Sur ce continent, les OGM ont pour vocation de se substituer à la base de l'alimentation, et non à apporter un produit à valeur ajoutée pour le consommateur.

Contrairement au nucléaire (qui reste pour une part contesté, mais aussi pour une part plébiscité), aux révolutions informatique, électromagnétique (portable, wifi) et numérique (dont on pointe régulièrement les limites, mais que l'on adopte très largement), et à la chimie (qui caractérise le développement technologique du second vingtième siècle), nous assistons pour la première fois dans l'histoire moderne, avec les biotechnologies, à un refus catégorique, massif et durable d'une innovation technologique pourtant initialement destinée à connaître une très large dissémination. Il faut dire que nous sommes confrontés à la première pollution vivante auto-multiplicative. Les promoteurs de cette technologie qui devait révolutionner les pratiques agricoles, mais aussi alimentaires, annonçaient triomphalement à la fin de la décennie quatre-vingt-dix que la majeure partie de l'Europe serait couverte d'OGM «dans dix ans». Or les OGM en Europe ne font au final que 0,05 \% des surfaces cultivées, soit de l'ordre de cent fois moins que l'agriculture biologique par exemple, après que les firmes biotechnologiques aient dépensé des millions de dollars en communication (souvent mensongère) et produit un incessant travail de lobbying auprès de l'Europe et des États membres,
\end{abstract}




\section{Open science et marchandisation des connaissances}

contrairement aux USA où sont cultivés $70 \%$ du soja et $25 \%$ du maïs transgénique. Plus innovante encore est la radicale nouveauté des formes d'organisation de la société civile en Europe pour faire face à cette politique d'innovation conçue comme une menace, et aux différentes conséquences qu'elles a engendrées sur la Science, sur les institutions en charge de l'expertise des risques, ainsi que sur la production de nouvelles formes d'expertise pilotées par la société civile. La production scientifique, à la fois comme connaissance et comme le monde dans lequel nous vivons, est donc questionnée, et même désormais coproduite, par des acteurs extérieurs à la l'institution scientifique, au marché ou à l'état, à commencer par les citoyens. Ceci nous amènera à interroger d'une part, la nature de la «science» en question, sa finalité et son mode de production, et d'autre part, les nouvelles formes de prises de participation citoyennes (qui peuvent être littéralement scientifiques) dans la régulation des nouvelles technologies en Europe.

Un concept central logé au cœur de la problématique de la participation dans les sciences, ou encore de la «science participative» doit faire l'objet d'une mise au point préalable, celui du risque technique et environnemental. Il revient aux sciences humaines d'étudier la manière dont la catégorie de risque, qui n'est qu'une manière parmi d'autres de dire l'incertitude, façonne nos institutions, les politiques publiques, le contexte législatif ou encore les attentes de la société civile. Depuis la publication de l'ouvrage de M. Callon et P. Lascoumes, Agir dans un monde incertain, l'idée de démocratie technique, ou de démocratie participative, a fait son apparition dans le vocabulaire des gestionnaires du risque technique et industriel. S'il est irréfutable qu'un certain nombre de «nouveaux risques» ont émergé dans le contexte de production d'innovations technologiques à caractère problématique (nucléaire, biotechnologies agricoles, nanotechnologies et bientôt, convergence des nano et des biotechnologies avec les technologies de l'information), la question de la régulation sociale et politique des produits de la science et de la technique n'est pas nouvelle; elle est même inhérente à la diffusion des produits de l'activité rationnelle. C'est donc dans un contexte de nouvelle organisation des enjeux institutionnels concernant la Science, la technique, l'État, l'économie et la société civile que nous avons analysé les modalités de participation de la société civile à «la Science», de la recherche fondamentale jusqu'aux différents niveaux de son application dans la réalité. 


\section{OGM et société, les raisons d'un divorce}

Aucune des plantes génétiquement modifiées sur 58,7 millions d'hectares en 2002 n'est génétiquement complètement connue. Des mutations des constructions génétiques artificielles ont été démontrées dans tous les végétaux étudiés. Plus de 99 \% de ces OGM sont essentiellement soja et maïs pour 83 \%, puis coton et colza. Six à huit produits alimentaires transformés sur dix peuvent contenir comme additifs courants de la lécithine de soja ou de l'amidon de maïs. La biologie moléculaire qui permet ces méthodes est la plus jeune des sciences de la vie, née en 1953 avec la découverte de la structure de l'ADN et dans les années 80 pour les modifications génétiques, et elle manque de recul pour savoir modifier sans risque les écosystèmes. En fait, la réglementation est issue au niveau mondial d'un concept de non-identification particulière des OGM, qui avait été imaginé dans les années 80 par les compagnies pour les banaliser. Ce manque d'identification résultait de l'application du principe $\mathrm{d}^{\prime}$ «équivalence en substance», notion au caractère plus politique que réellement scientifique qui postule qu'une plante génétiquement modifiée est, par sa composition chimique, globalement équivalente à sa version non OGM. L'Europe a cependant choisi, préalablement à la libéralisation éventuelle des OGM, de mettre en place traçabilité, étiquetage, moyens de contrôle et d'évaluation scientifiques sérieux, c'est-à-dire des directives et règlements spécifiques, ainsi qu'une agence de sécurité sanitaire des aliments. Il est clair que l'on ne peut pas réaliser des progrès pendant quarante ans en agro-alimentaire sans savoir dire exactement ce que la population consomme, sans reconnaître précisément les produits en cas d'accident. Il restera à travailler sur l'organisation de l'expertise indépendante et contradictoire, mais ceci est un autre chantier indispensable. Tout ce contexte a été douloureux pour de nouvelles autorisations. D'autant que trois axes différents se sont entrechoqués dans tous les débats sur les OGM: d'abord, il y a eu des discussions autour de la révolution culturelle sur le franchissement systématisé et industrialisé de la barrière entre les espèces vivantes, ensuite on a remis en cause l'évaluation scientifique négligée notamment par une forte volonté de promouvoir les biotechnologies, laquelle a été jugée accouplée à des lacunes de formations dans les sciences de l'environnement et la toxicologie. Troisièmement, les pressions techno-économiques avec les brevets sur les gènes et le vivant associés aux OGM ont été hautement discutées.

Cependant, la majorité des intervenants semble convenir qu'arrêter toute recherche ne serait pas sérieux. En fait, comme il a été développé dans Ces 


\section{Open science et marchandisation des connaissances}

OGM qui changent le monde ${ }^{1}$, deux stratégies s'offrent pour qui décide d'expérimenter de nouveaux OGM aux champs. La première, celle qui a été suivie, et a conduit à des révoltes paysannes et écologistes, et à des arrachages illégaux, consiste à trier en premier lieu en pleine terre tous les OGM obtenus sur des critères agronomiques, puis ensuite à commencer quelques tests de toxicologie dite aiguë avec le petit nombre des OGM élus. Par exemple, la première question est avant tout de savoir si ces plantes peuvent vraiment bien vivre en présence de désherbant, produire la protéine insecticide ou médicamenteuse voulue issue de chien, de bactérie ou d'homme, etc. On fauche alors et enterre ce qui ne marche pas, la vaste majorité des essais. On va loin, on hybride ensuite les OGM les plus réussis aux meilleures variétés commerciales, avant tout essai de toxicité. Il serait par contre possible de trier les premiers OGM en serre, et d'exiger des résultats préalables, puisqu'il est admis que toute diffusion dans l'environnement ne peut pas être strictement étanche. Trois raisons sont principalement évoquées pour s'opposer à ce dernier choix: il est nécessaire de fabriquer beaucoup d'OGM afin que très peu fonctionnent vraiment, or la technique n'en est qu'à ses débuts, et la complexité des interactions génétiques n'est pas gérable avec le niveau de connaissances actuel. De nombreux tris en serre reviendraient donc trop chers pour rentabiliser cette technique par rapport à des solutions concurrentes: gérer différemment les pesticides, produire les protéines par des bactéries ou des levures au laboratoire, comme on fait pour l'insuline... Deuxièmement, il est répété que les plantes se comportent différemment aux champs et en serre, ce qui souligne le caractère imprévisible des régulations génétiques complexes. Dans cette histoire en effet, les essais d'adaptation au gel ou à la sécheresse par exemple ne sont vraiment pas légions: on ne sait pas encore avec un seul gène répondre à une situation environnementale variable, et peut être que l'on apprendra bientôt que cela n'est pas possible de manière aussi simple. Troisièmement, la logique de l'économie scientifique et de l'économie industrielle bouscule ensemble la logique de la protection de l'environnement; mais comment ne pas reconnaître là un défaut général attribué à notre société productiviste? Convient-il alors, comme plus de 1300 maires voulant protéger leur agriculture l'ont demandé en France, de rayer ces essais de la carte? Tout le monde pourra-t-il être concilié un jour?

Là se justifie mieux la nouvelle stratégie possible. Certains tests de toxicologie pour les hommes et les insectes sont envisageables bien avant de sortir de la serre: il est concevable de les réaliser quitte à les compléter ensuite,

1. Séralini G.-E., Ces OGM qui changent le monde, Flammarion, coll. Champs, 2004. 
puisque les champs des agriculteurs ne pourront jamais être acceptés socialement comme servant de paillasses aux scientifiques. La castration des plantes potentiellement toxiques sortant aux champs, les filets anti-insectes, l'incinération des déchets et racines, tout cela pourrait être la règle et non l'exception comme au $\mathrm{xx}^{\mathrm{e}}$ siècle. Les objectifs des recherches pourraient être publiés et choisis par un comité comprenant la société civile. En effet, aucun représentant des consommateurs n'a pu siéger pendant longtemps à la Commission du Génie Biomoléculaire, qui évaluait en France les OGM avant le Haut Comité des Biotechnologies, malgré les recommandations. Les contrôles des expérimentations sont normalement prévus pour être réalisés avec le sérieux et les moyens qui s'imposent: le décret permettant aux agents de l'État de prélever des échantillons dans les champs des industriels, pour vérifier si des plantes sont OGM, n'existe toujours pas; les méthodes pour doser les OGM expérimentaux par des laboratoires non plus, alors que depuis quinze ans des OGM ont été expérimentés sur les terres agricoles... Le risque est que ces lenteurs soient prises pour des hontes dans l'histoire des sciences. Certes, des projets risquent de ne pas voir le jour avec ces critères plus stricts. Mais n'est-ce pas à ce prix que souvent se développent les recherches de qualité pour l'environnement et l'agriculture?

\section{Risques sanitaires et environnementaux identifiés}

Le risque lié aux protéines insecticides produites par $25 \%$ des OGM commercialisés dans le monde vient $d u$ fait que ces insecticides synthétiques nouveaux, fabriqués directement dans les cellules de plantes consommables, n'ont jamais été homologués ainsi que des produits phytosanitaires courants, et notamment au niveau de leur toxicité sur mammifères. Des subtilités juridiques ou réglementaires ont permis ce contournement de l'évaluation. Par exemple, l'éclatement des globules rouges humains in vitro en présence de la protéine tronquée et/ou mutée provenant de certains maïs OGM pourrait être demandé à l'étude, car ce phénomène est montré pour une protéine $\mathrm{Bt}$ tronquée. L'absence d'effet sur les échanges électrolytiques ou la porosité des parois intestinales de mammifères devra être documentée dans le futur. Les protéines Bt utilisées ont ce type d'action chez des insectes ciblés.

Les risques liés aux herbicides tolérés par les OGM, ou des produits issus de leur métabolisme nouveau par la plante transgénique, ou par l'animal s'en 


\section{Open science et marchandisation des connaissances}

nourrissant, ainsi que les risques liés aux adjuvants et à leurs produits de transformation associés à ces herbicides, ont posé des problèmes hormonaux, de reproduction, nerveux, immunitaires et de mutations à moyen et long terme chez les mammifères. En effet, cette question n'a jamais été traitée auparavant pour les herbicides associés aux OGM, notamment car les plantes traitées avec ce type de désherbants étaient en général détruites et non consommées. Or, des herbicides peuvent être absorbés en grandes quantités par les plantes tolérant un herbicide (75\% des OGM).

Le risque lié à l'utilisation de séquences virales introduites dans la plupart des OGM a été évoqué. Il n'est pas pris en compte dans sa composante temporelle et évolutive. Les recombinaisons génétiques et la susceptibilité des plantes aux infections virales pourraient être favorisées par ce type de séquences, ou la création de nouveaux virus.

Le risque de développement des allergies: des tests cutanés pour détecter les allergies éventuelles à tous les nouveaux OGM pourraient être mis sur le marché afin de prévenir ce risque, tout du moins pour les OGM rentrant par leurs produits dérivés dans une vaste majorité d'aliments. Ils ne sont cependant pas prévus. L'étiquetage systématique a été la meilleure réponse dans un premier temps.

Le risque des gènes marqueurs de résistance aux antibiotiques: des morts étant déjà dues à la dissémination incontrôlée des gènes de résistance aux antibiotiques par infections résistantes, et à la mauvaise consommation d'antibiotiques, il a été convenu que les OGM contenant des gènes de résistance aux antibiotiques devaient être retirés des catalogues officiels des semences et interdits à la culture, même expérimentale, après 2008. Leur rémanence importante a constitué le symbole de la négligence dans l'évaluation des OGM.

Les risques de désordres métaboliques causés directement ou indirectement par un OGM, plante ou micro-organisme, peuvent être liés à l'insertion aléatoire du transgène, ou aux séquences surnuméraires inutiles de la construction génétique artificielle, perturbant le métabolisme de l'OGM de manière non facilement détectable par l'analyse de l'équivalence en substance. Ils sont donc à évaluer par des tests de longue durée de nutrition et de toxicologie préalables sur mammifères. Ceux-ci n'ont jamais été réalisés par les entreprises pour les dossiers d'autorisation des OGM au-delà de trois mois, le plus souvent en deçà ${ }^{2}$. Cela demeurera pour les quinze premières années de consommation des OGM, destinés à contenir de nouveaux pesticides, une honte dans l'histoire de la science, alors que les autorités gouvernementales

2. Séralini G.-E., Nous pouvons nous dépolluer, Ed. J. Lyon, 2009. 
voudraient convaincre le monde entier (y compris les plus vulnérables) qu'il est sain ou sans risque d'en manger pendant sa vie entière.

Quant aux risques des micro-organismes génétiquement modifiés ou MGM, la directive européenne 98/81/CE modifiant la directive 90/219/CEE relative à l'utilisation confinée de MGM exclut de son champ d'application certaines techniques telles que la mutagenèse et l'autoclonage. Cette formulation signifie que les MGM au sens législatif ne couvrent qu'une partie des MGM au sens scientifique: quelle que soit la qualité des constructions génétiques réalisées, la perte objective du sens du terme «MGM» conduit, d'une part, à l'impossibilité de toute traçabilité créant donc de nouveaux risques alimentaires, et, d'autre part, à la perte de la liberté de choix pour le consommateur, et ce, dans les deux cas, par absence d'obligation d'étiquetage.

Sur l'environnement, les risques de dissémination et croisements (par des transferts génétiques dits par les spécialistes verticaux et horizontaux) sont à l'étude chez des plantes, animaux et micro-organismes génétiquement modifiés dans l'environnement. En effet, les modifications génétiques importantes du vivant dans les conditions naturelles ne sont ni rattrapables ni recyclables, et les assurances n'ont pas pris le risque OGM en charge.

Notons que $99 \%$ des caractères introduits chez les OGM sont des caractères d'envahisseurs (production d'insecticide ou capacité à vivre en présence de désherbants communs polluant champs, bords de routes ou voies ferrées) et qu'en ce sens ils remettent en cause la biodiversité des écosystèmes.

Les risques de contamination des semences ou des récoltes non OGM par des traces d'OGM ont déjà été avérés. L'idée d'une banque de données où les OGM, même expérimentaux, pourraient voir leurs séquences déclarées à un registre public afin de mettre en œuvre leur détection est à l'étude. De même, les contaminations de la chaîne alimentaire pourraient être prises en charge par les propriétaires des événements de transformations génétiques concernés. De claires séparations de filières sont mises en place dans le monde.

Les risques induits par l'application d'insecticides ou d'herbicides en excès par rapport aux prévisions, même sur les champs contaminés ou les zones non agricoles, pour gérer les disséminations d'OGM, ou d'insectes liés aux OGM, sont importants. 


\section{Un déficit structurel d'expertise publique face à des risques avérés}

Les OGM constituent des outils pour simplifier les épandages de pesticides à court terme et nourrir les vaches des pays riches. Ils sont physiquement associés au développement de l'agriculture intensive, et les sojas et maïs OGM n'ont ni aboli ni réduit globalement l'usage des pesticides en Amérique du Nord, contrairement à leurs arguments publicitaires. D'ailleurs, un débat est ouvert pour savoir si une stratégie qui fabrique des plantes nouvelles, capables de s'imbiber d'un produit qui leur était toxique, un désherbant, sans les faire mourir, peut faire partie d'une vision d'agriculture durable. Les sojas au Roundup conçus par la Société Monsanto sont les OGM majoritaires dans le monde et exportés dès 1996. Les autorisations de cultures de variétés de maïs transgénique à propriété insecticide ont déjà été largement accordées depuis 1997 sur le marché européen, et elles sont surtout exploitées jusqu'en 2009 seulement par une petite partie de l'Espagne agricole. Celles-ci n'ont pas été suffisamment valables au niveau agronomique, ni efficaces, ni valorisées pour la plupart des agriculteurs européens. Ces derniers se sont donc imposés de fait leur propre moratoire depuis 1998, date des premières libérations commerciales des cultures, et ceci indépendamment des politiques.

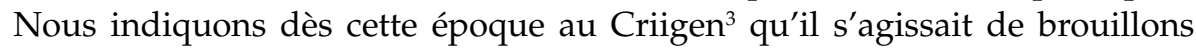
scientifiques mal faits avec gènes de résistance aux antibiotiques, mal évalués sans en nourrir des rats longtemps au préalable, et enfin mal étiquetés. Ils ont été échaudés, d'autant que ces OGM ne représentaient qu'une solution publicitaire, mais non efficace à un problème majeur, rendre l'agriculture plus durable.

3. Comité de recherche et d'informations indépendantes sur le génie génétique. Il s'agit d'un comité apolitique et non militant d'expertise, de conseil, indépendant des producteurs d'OGM, intervenant à différents niveaux, juridique, scientifique (santé, environnement), sociologique, technique (étiquetage), notamment pour des dosages d'OGM, et au niveau économique. 


\section{L'évaluation des biotechnologies par les sociétés civiles européennes : critères pertinents}

Les récents débats engagés au cours de l'année 1998 en France autour de l'euthanasie ont révélé, comme dans de nombreux autres domaines, l'existence de vides juridiques, qui agissent comme déclencheurs et moteurs d'un travail éthique et in fine politique sur les choix sociétaux à réaliser au sujet des nouvelles pratiques que nous autorise, en particulier, la mise à disposition de moyens techniques inédits. Il en est de même pour les OGM qui tendent à révéler aux sociétés l'existence de tels vides juridiques (comme dans le cas de la crise de la vache folle), mais aussi, et surtout, de «vides scientifiques » à l'égard desquels l'état actuel des connaissances ne permet pas d'apporter aux hommes des garanties suffisantes en matière de santé publique ou de qualité de leur milieu de vie. Ainsi, le problème anthropologique essentiel que posent les OGM réside dans le fait qu'ils échappent à nos sens, mais pas à nos représentations du risque inhérent à leur consommation. La question de l'étiquetage, comme production d'une trace de l'origine, à l'instar de la viande bovine depuis la crise de la vache folle, devient centrale au titre des solutions envisagées pour «gérer» ce qui émerge sous la forme d'une «crise». Nous avons bien là affaire, comme pour le nuage de Tchernobyl ou la maladie de la vache folle, à une nouvelle forme de contamination - réelle ou supposée, en l'occurrence prouvée pour les OGM - dont la caractéristique principale serait d'échapper à toute forme de reconnaissance par les sens dont est doté l'organisme humain. Impalpable, inodore, incolore et sans saveur, la modification génétique apparaît comme une menace potentielle inscrite au plus profond de l'organisme en question et est affectée d'un pouvoir contaminant, $c^{\prime}$ est-à-dire susceptible de modifier l'organisme qui entrera en contact avec la substance «contaminée». L'émergence tardive de la question de l'étiquetage en 1998, alors que les OGM sont mis en culture depuis 1996, est le signe manifeste d'un écart sans cesse grandissant entre la réglementation d'une part, et la vitesse de production et de mise en circulation des marchandises de l'autre. De ce point de vue, l'OGM peut apparaître comme infiniment plus redoutable que les contaminations bactériologiques ou virales traditionnelles. On ne peut pourtant ignorer l'alerte donnée par de nombreux scientifiques, qu'ils soient ou non mandatés en qualité d'experts pour évaluer les risques, comme d'autres avaient déjà et depuis de nombreuses années appelé à la vigilance au sujet de l'amiante, des rejets nucléaires dans l'environnement, accidentels ou non, et plus récemment de la maladie de la vache folle, avant que ces 


\section{Open science et marchandisation des connaissances}

problèmes n'émergent sous forme de «crises» dans l'espace public. C'est donc à l'appel d'organismes de recherche indépendants développés en open science afin d'être tournés vers la société civile tels que le CRIIGEN en France ou le CDG (Consiglio dei Diritti Genetici) en Italie, de lanceurs d'alerte individuels (le chercheur du Royaume-Uni Arpad Pulzei, le français Christian Vélo du CRIIGEN), et d'organes de diffusion d'information indépendants (Inf'OM en France), que les acteurs des sociétés civiles européennes ont répondu. Le CRIIGEN a, depuis 1999, joué un rôle moteur sur le plan international dans la contre-expertise des OGM, comme le groupe ENSSER fondé en 2009, réseau européen de scientifiques concernés et socialement responsables, fondé avec C. Vélo et G.-E. Séralini. Il est en relation avec l'Union of Concerned Scientists aux États-Unis. Qu'ils soient chercheurs, bien entendu, mais aussi producteurs, distributeurs, restaurateurs, agriculteurs, responsables de coopératives... ou tout simplement citoyens et consommateurs, de nombreux acteurs ont décidé de ne pas abandonner le travail scientifique (de la production de connaissances à la «fabrication» du monde) aux seuls organismes d'État ou aux multinationales. Connues en France sous le concept de «science citoyenne», ces nouvelles formes d'implications des organisations issues de la société civile prennent des formes variées, comme le sont les modes d'action, les attentes et les analyses qu'elles développent.

\section{Open science ou science participative?}

L'analyse de nombreuses expériences de participation à science dans sept pays d'Europe atteste de l'existence de nombreuses difficultés dans sa mise en œuvre, notamment lorsque cette dernière est financée par des fonds publics. En revanche, lorsque l'action participative est initiée en dehors du cadre institutionnel et réglementaire, elle est souvent perçue par les industriels et les autorités comme un blocage au "progrès technique», bien que la majeure partie des organisations issues de la société civile (OSC) qui sont engagées dans les débats sur le développement des biotechnologies agricoles ne soient pas «antiscience». La recherche que nous avons menée pour le compte de la Communauté européenne nous a, en effet, permis de confirmer le fait que la science est toujours le résultat d'un processus de co-production dans lequel plusieurs acteurs contribuent à définir les connaissances pertinentes. Mais un sentiment d'inégalité demeure, notamment du côté des organisations citoyennes et nombreuses sont les OSC qui estiment que l'actuel cadre institutionnel de prise de décision est orienté de manière disproportionnée vers la coopération entre la science et l'industrie, au détriment des intérêts des citoyens (prise en compte des risques sanitaires et écologiques avérés 
ou potentiels, création d'une nouvelle arme alimentaire par la brevetabilité $\mathrm{du}$ vivant, etc.). Les associations en question estiment qu'il est nécessaire de réexaminer la façon dont la «science» alimente l'innovation, de sorte que l'ensemble du processus soit transparent et plus équitable au sens où le processus d'innovation apparaît comme une privatisation des bénéfices de la recherche publique et une socialisation des risques inhérents. De plus, les citoyens perdent confiance dans les organismes officiels d'évaluation qui, globalement, depuis quinze ans, se sont comportés en vassaux de l'industrie en accordant aveuglément et servilement des autorisations, et en assumant, malgré les demandes de transparence réitérées, le fait de baser leurs décisions sur des données sanitaires tenues secrètes à la demande des industriels. De plus, ces tests sanitaires ne sont réalisés que par les compagnies qui conçoivent et commercialisent les OGM. Cette politique pour le moins obscurantiste nécessite, on l'aura compris, une révision générale des procédures d'évaluation et donc une nouvelle autorité politique, au risque d'entraîner une aggravation de la crise qui traverse les technosciences si l'on en juge la méfiance avec laquelle sont accueillies les nanotechnologies.

La concertation en amont de la société civile pourrait, en outre, permettre la mise en évidence de certains problèmes avant que d'énormes investissements publics soient réalisés. La concertation, dans la mesure où elle n'est pas de pure forme, permettrait, toujours selon les citoyens, d'enrichir la démarche d'innovation en raison de l'élargissement de la gamme d'expériences prises en compte, celle des chercheurs, mais aussi des producteurs, des distributeurs et des consommateurs. Il est enfin suggéré qu'une articulation plus étroite entre science, société et innovation faciliterait l'action responsable et renforcerait la confiance mutuelle entre ceux qui collaborent pour mettre au monde ces produits d'une manière plus durable.

Par conséquent, il apparaît d'autant plus urgent de précéder à une redéfinition de la «participation à la science » proposée par les OSC, en impliquant la société civile à un stade précoce dans le processus de recherche, c'est-à-dire en amont du processus de décision. La participation aux étapes réglementaires du processus de la science et de l'innovation ne peut selon elles advenir que si la société civile est associée dès la mise en place de l'agenda. Or, presque tous les événements et les procédés mis en œuvre pour renforcer la participation sur invitation ont été jugés insatisfaisants et le point de vue général est trop peu d'efforts ont été produits pour faire fonctionner les systèmes participatifs, jugés trop bureaucratiques. Les OSC regrettent également un manque, au sein des organismes institutionnels, de "capacité » de prise en compte des résultats de cette participation élargie. Ce manque, qui peut être compris comme une carence institutionnelle, mais le plus souvent comme une stratégie du pouvoir, 
amène de nombreuses parties prenantes à envisager d'autres modalités de participation, plus autonomes, allant jusqu'à la perspective de mise en œuvre de formes de désobéissance civile et civique (fauchage volontaire, interdictions d'OGM prises par certains élus, etc.). La participation «non-invitée», plus immédiate, vise l'ouverture du débat et de la création d'opportunités permettant aux OSC de faire entendre leur point de vue: dans les tribunaux, dans les batailles juridiques, dans les boycotts de consommateurs et dans les campagnes médiatiques. Elle apparaît bien comme une forme universaliste de construction d'un espace politique de négociation, loin de la conception localiste qu'on a pu lui prêter, plus connue sous appellation de syndrome NIMBY (littéralement: pas dans ma cour).

\section{Principales propositions pour améliorer la participation sur invitation (au niveau institutionnel)}

En guise de conclusion, et au terme de l'enquête européenne, il est ressorti un panel de propositions émanant des OSC en vue d'améliorer les processus de science participative. La liste suivante n'est ni exhaustive (l'enquête ne concernait que sept pays), ni classée par ordre de priorité:

- L'UE, ainsi que les institutions scientifiques et politiques devraient fournir aux OSC des espaces et des opportunités pour exprimer leur opinion et influencer le processus dans un débat ouvert.

- Les possibilités de participation devraient exister dès le début du processus, lorsque les projets de recherche sont au stade du financement ou de l'autorisation.

- Élargir l'objet du débat pour y inclure les implications sociales et politiques, et non pas seulement les questions techniques, sanitaires et économiques.

- Promouvoir la transparence en matière de politique scientifique. Le public devrait être informé de ce que sont les objectifs ultimes de la recherche financée sur fonds publics, des intérêts en jeu et des risques impliqués.

- Mettre l'accent sur une approche précautionneuse qui tient compte des incertitudes scientifiques et des possibles risques et conséquences sur le long terme, par une expertise pluridisciplinaire alternative et transparente. 
- Garantir le débat public, mais aussi la prise en compte des conclusions de ces débats.

- Ouvrir le processus d'innovation afin de s'assurer que tout le monde, particulièrement les principales parties prenantes comme les agriculteurs, a suffisamment de temps et de possibilités d'examiner les implications des nouveaux produits et d'exprimer leur opinion sur les questions importantes.

- La contre-expertise est considérée comme la meilleure façon de révéler que les experts nommés par le pouvoir peuvent avoir un parti pris en faveur de l'industrie. Cependant, il ne peut pas y avoir de contreexpertise sans financement. Le financement de la contre-expertise est considéré comme un moyen d'atteindre une plus grande objectivité sur les problèmes liés aux objectifs de la recherche et aux applications techniques.

- Les questions d'ordre général, telles que l'adoption de technologies très innovantes, pourraient être soumises à plus de démocratie directe (par exemple un référendum).

De plus, à un niveau plus large (niveau sociétal):

- Promouvoir la recherche qui répond aux besoins des agriculteurs telle l'agriculture biologique en tant que mode de développement alternatif, durable et économiquement viable.

- Promouvoir la coopération dans des projets de recherche entre les OSC et les institutions scientifiques, comme dans le cas des PICRI (Partenariat Instituts Citoyens pour la Recherche et l'Innovation) et du CRIIGEN.

- Diffuser l'information et améliorer l'éducation scientifique à tous les niveaux, en organisant des conférences et en créant des espaces adéquats pour un dialogue avec la société civile.

- Promouvoir la transparence sur les tests sanitaires et environnementaux effectués par l'industrie, dès l'obtention des données brutes. 


\section{0 principes pour une participation efficace}

- Le financement de la recherche scientifique doit être alloué en fonction de l'intérêt public et des besoins de l'utilisateur final.

- La participation précoce de la société civile, à un méta-niveau, lorsque que les termes du processus d'innovation sont non-techniques.

- Tout le monde pourrait, et devrait, être capable de participer à un certain niveau et dans une certaine mesure, ce qui devrait inclure les OSC en tant que parties prenantes.

- La participation doit se faire sur un pied d'égalité pour éviter les relations de pouvoir inégalitaires.

- L'échange d'information dans les deux sens, l'ouverture d'esprit et un engagement véritable, de la part des institutions scientifiques, entre elles-mêmes et avec les citoyens, notamment au niveau des tests sanitaires effectués, mais aussi sur les méthodes d'obtention des nouveautés et leurs essais préalables à la commercialisation.

- Les débats sur la science devraient impliquer différents point de vue/ opinions, une pluralité d'expertises et la reconnaissance d'autres types de connaissances qui prennent en compte l'opinion des minorités.

- L'ouverture et la transparence sont cruciales dans le développement, la pratique et la régulation de la recherche financée sur fonds publics.

- Une information facilement accessible et non-technique est requise. Le public doit avoir la possibilité d'acquérir une bonne compréhension des questions techniques.

- La participation à la science exige de tenir compte d'intérêts et de modes de vie spécifiques, par exemple le point de vue et les exigences spécifiques des femmes et les besoins et le calendrier des agriculteurs.

- La participation du public à la science exige que la preuve soit faite que les préoccupations du public ont été écoutées et prises en compte. 